\title{
Neurosarcoidosis: guidance for the general neurologist
}

\author{
Neurosarcoidose: orientações para o neurologista geral \\ Livia Almeida Dutra, Pedro Braga-Neto, Ricardo Araújo Oliveira, José Luiz Pedroso, Agessandro Abrahão, \\ Orlando Graziani Povoas Barsottini
}

\begin{abstract}
Neurosarcoidosis (NS) more commonly occurs in the setting of systemic disease. The diagnosis is based on a clinical history suggestive of NS, presence of noncaseating granulomas, and supportive evidence of sarcoid pathology, laboratory, and imaging studies. NS could involve any part of the nervous system and often demands high doses of steroids for symptom control. It presents low response to isolated steroids administration and frequently requires immunosuppressive agents. In NS, lymphocytes are polarized toward an excessive Th1 response, leading to overproduction of TNF-alpha and INF-gama, as well as IL-2 and IL-15. Infliximab, a chimeric monoclonal antibody that neutralizes the biological activity of TNF-alpha, is a new option in the NS treatment. We revised pathophysiology, clinical manifestations, diagnostic work up, and treatment of NS as guidance for the general neurologist.
\end{abstract}

Key words: sarcoidosis, neurosarcoidosis, methotrexate, azathioprine, cyclophosphamide, infliximab.

\section{RESUMO}

A neurosarcoidose (NS) ocorre frequentemente no contexto de doença sistêmica. O diagnóstico é baseado na história clínica sugestiva de NS, presença de granulomas não-caseosos e achados anatomopatológicos, laboratoriais e radiológicos de sarcoidose. A NS causa manifestações neurológicas variadas, que apresentam, em geral, baixa resposta ao corticoide isoladamente e, portanto, necessitam uso de imunossupressores. Na NS, os linfócitos estão polarizados para resposta Th1 excessiva, levando à produção aumentada de TNF-alfa e IFN-gama, assim como IL-2 e IL-15. Infliximabe, um anticorpo monoclonal quimérico que neutraliza a atividade biológica do TNF-alfa, é uma nova opção no tratamento da NS. Revisou-se a fisiopatologia, as manifestações clínicas, o diagnóstico e o tratamento da NS para orientar neurologistas gerais.

Palavras-Chave: sarcoidose, neurosarcoidose, metotrexato, azatioprina, ciclofosfamida, infliximabe.

Sarcoidosis is an inflammatory multisystem disorder of unknown etiology with a worldwide distribution that can affect lungs, lymphatic system, skin, liver, eyes, and nervous system ${ }^{1}$. We owe the first recorded and illustrated description of sarcoidosis to Jonathan Hutchinson in 1878, when he reported a patient with a dermatologic disease, which comprised purplish, symmetric, and non-tender skin plaques, initially considered a manifestation of gout ${ }^{2}$. The term sarcoidosis comes from histological studies performed by Cesar Boeck, in 1899, who described pathological findings of skin lesions that resembled those of sarcoma, which he called 'sarkoid' or 'sarcoma-like'. Involvement of the nervous system was first recognized, in 1905, by Winkler, and, in 1909, Heerfordt described three patients in the context of facial nerve palsies, uveitis, parotid enlargement, and fever ${ }^{1,3}$.

Neurosarcoidosis (NS) may involve any part of the nervous system with acute and chronic courses ${ }^{1,4}$. Cranial nerves, hypothalamus, and pituitary gland are the most commonly regions involved, but meninges, parenchyma, brainstem, and spinal cord may also be affected ${ }^{4}$. Clinical differentiation from other neurological diseases may be difficult when an isolated involvement of the nervous system is present ${ }^{3,4}$. In this review, we highlighted its clinical forms, pathogenesis, and treatment guidelines for NS, as guidance for general neurologists.

\section{NEUROLOGICALMANIFESTATIONS OFSARCOIDOSIS}

Neurologic manifestations are found in 5 to $20 \%$ of cases and symptoms may be mild or even severe, requiring a more aggressive intervention. About one-half of patients with NS can develop neurologic manifestations before systemic sarcoidosis is evident ${ }^{5}$. 


\section{Hydrocephalus and chronic meningitis}

Meningitis may present as a single episode or as a recurrent or chronic course. Cerebrospinal fluid (CSF) pleocytosis often occur in the setting of other manifestations, such as cranial neuropathy, myelopathy, mass lesions, or other focal neurologic manifestations. Granulomata in the fourth ventricle could be responsible for hydrocephalus, which may require urgent treatment ${ }^{6}$.

\section{Central nervous system}

NS patients with headache may present meningitis, increased intracranial pressure or granulomatous mass lesions, which may mimic demyelination or neoplasm lesions ${ }^{7}$. In patients with intraxial lesions, seizures are found in 5 to $22 \%$, including generalized, complex partial and myoclonic seizures $^{8}$. Hypothalamic and pituitary involvement is a characteristic feature of NS, and the posterior pituitary is more commonly affected than the anterior. Polydipsia and polyuria may be the presenting symptoms of NS secondary to diabetes insipidus. Other manifestations include hypothyroidism, amenorrhea, galactorrhea, sleep disorders, weight gain, temperature dysregulation, and personality changes. The possibility of endocrine dysfunction should always be investigated in the context of nonspecific complaints such as weight loss, fatigue and generalized weakness 9 .

Patients with NS may present white matter abnormalities on the magnetic resonance imaging (MRI) (Fig 1) and progressive multifocal leukoencephalopathy should be considered as a differential diagnosis ${ }^{10}$. Myelopathy in NS is unusual, often evolving over weeks to months, with radiculopathy
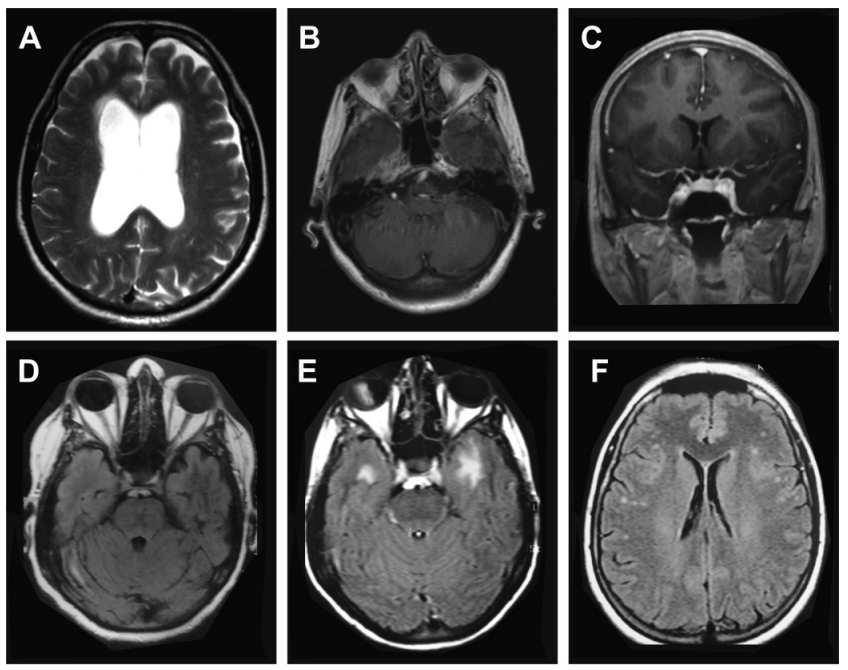

Fig 1. Brain magnetic resonance images of patients with neurosarcoidosis. A: hydrocephalus; B: meningeal thickening involving sphenoid wings and temporal lobes; C: meningeal thickening involving cavernous sinus with gadolinium enhancement; D: involvement of the right cerebellar hemisphere; E: leukoencephalopathy of anterior temporal lobes; F: white-matter hyperintensities in cortico-subcortical regions of frontal lobes suggestive of vasculitis. or long tract dysfunction. Intramedullary sarcoidosis may present focal enlargement of the cord with gadolinium enhancement. Progressive motor dysfunction may even mimic anterior horn syndrome ${ }^{10}$.

Involvement of basal ganglia may lead to movement disorders, with rare reports linking sarcoidosis to Parkinsonism and generalized chorea ${ }^{11}$. Stroke, subarachnoid hemorrhage, or intraparenchymal hemorrhage are quite rare in NS and may result from blood vessels involvement by sarcoid granulomas ${ }^{9,11}$. Most NS patients with increased intracranial pressure present mass lesions, meningitis, or venous sinus thrombosis. In despite of that, few reports described pseudotumor cerebri due to $\mathrm{NS}^{12}$.

\section{Neuropsychiatric manifestations}

Patients with sarcoidosis and extensive central nervous system (CNS) involvement may develop dementia, psychoses, depression, poor concentration, hallucinations, and encephalopathy. Neuropsychiatry symptoms (NPS) develop months, sometimes presenting a relapseremitting course. They can be found in sarcoid patients without evident CNS involvement. However, when NPS are found, CSF analysis is frequently abnormal, disclosing pleocytosis or increased protein ${ }^{13}$.

\section{Cranial and peripheral neuropathy}

The most common cranial neuropathy in NS is facial palsy, which may represent an isolated manifestation or which can occur in the context of multiple cranial nerve involvement. NS should be considered when multiple cranial nerves are affected or in patients with bilateral facial palsy. The optic nerve is involved in up to $38 \%$ of NS patients and some may present a progressive and painless monocular visual loss, sometimes with papilledema or optic atrophy. Less often, an acute or subacute visual loss may occur. Retrobulbar optic neuritis mimics multiple sclerosis. Approximately $20 \%$ of patients have uveitis, usually bilateral, granulomatous that may be anterior (more common) or posterior ${ }^{14,15}$.

Sarcoid patients may present hearing loss or vestibular dysfunction due to acoustic neuropathy. Lower cranial nerves are more affected than those responsible for extraocular movements (III, IV, and VI), determining dysphagia or dysarthria ${ }^{16}$.

Involvement of the peripheral nerves is a well-recognized feature of NS, occurring in up to $20 \%$ of patients. Most of them present depressed tendon reflexes or minor sensory changes, although severe weakness may be found. The clinical presentations mainly include symmetrical distal neuropathy, mononeuropathy, mononeuritis multiplex, and acute or chronic demyelinating polyneuropathy. The mechanism of nerve injury is unclear, with evidence for both axonal injury and demyelination process. Considering that some NS patients have presented chronic inflammatory demyelinating polyneuropathy, 
some authors believe that cytokines and immune factors may also play a role in nerve injury ${ }^{17}$. Peripheral neuropathy may be associated with myositis or CNS involvement.

\section{Myopathy}

Asymptomatic involvement of the skeletal muscle by sarcoid granulomas may occur in more than $50 \%$ of patients with sarcoidosis. Only $1-2 \%$ of the patients present symptomatic muscle disease. Three distinct myopathy clinical presentations are recognized: acute myositis, nodular form, and chronic form. The most common of them is the chronic form, which presents slowly progressive weakness and normal creatine phosphokinase levels, whereas acute myositis is rarely found. Patients with the nodular form may present palpable nodules. MRI and muscle imaging with gallium scanning provide more information for establishing diagnosis; however, muscle biopsy may be required for diagnosis ${ }^{9,11}$.

\section{PATHOPHYSIOLOGY}

The etiology of NS is multifactor, involving genetic predisposition, environmental and individual factors. The ACCESS study demonstrated that agricultural employment, mold or mildew, musty odors at work, and pesticide-using industries are modestly associated with sarcoidosis risk $(\mathrm{OR}=1.5)^{18-20}$. On the other hand, tobacco was negatively associated with sarcoidosis ${ }^{18-20}$. Research into specific microbial etiologies showed that mycobacterial DNA and protein antigens are present in sarcoidosis tissues and are the target of $\mathrm{T}$ and $\mathrm{B}$ cell responses, suggesting some important etiologic factors ${ }^{18}$. It is speculated that deposition of microbial antigens and insoluble particules aggregates with specific host proteins in granulomas, generating an antigenic stimulus, followed by T-cell and macrophage activation via a classic major histocompatibility complex (MHC) II-mediated pathway ${ }^{21}$.

The lymphocytes are polarized toward an excessive Th1 response, leading to overproduction of TNF-alpha and INF-gama, as well as $1 \mathrm{~L}-2$ and IL-15. The production of IL-12 and 18 also is increased, which enhances IFN-gama production and induces differentiation of Th0 precursors into active Th1 lymphocytes, perpetuating the response ${ }^{21}$. The CD4+ lymphocytes are central to the pathogenesis of sarcoidosis, triggering the granuloma formation ${ }^{18}$. These basic immunologic features (Th1 polarization, oligoclonal T-cell expansions) are found in patients with sarcoidosis, despite clinical manifestations.

Genetic linkage studies have identified the butyrophilin-like 2 gene (BTNL2), localized in the MHC region and involved in T-lymphocyte activation and regulation, as a susceptibility factor for the development of sarcoidosis ${ }^{18,19}$. Human leukocyte antigens (HLAs) DR subtype of class 2 antigens is associated with many inflammatory conditions, including sarcoidosis. HLA-DRB1*03, HLA-DRB1*11, HLA$\mathrm{DRB1}^{*} 12$, HLA-DRB1*14, and HLA-DRB1*15 have been associated with an increased risk of developing the disease. HLA-DRB1*03 has been found to be associated with Löfgren syndrome, an acute form of sarcoidosis ${ }^{19}$.

\section{DIAGNOSTIC WORK UP}

NS should be suspected in patients with documented sarcoidosis that present neurologic complaints. In 50\% of the cases, neurological symptoms begin at the first signs of systemic disease $^{22}$. The diagnosis is based on evidence of a clinical history suggestive of NS, exclusion of other conditions capable of producing noncaseating granulomas, presence of noncaseating granulomas, and supportive evidence of sarcoid pathology, laboratory and imaging studies ${ }^{15}$. Zajicek et al. established a classification to the diagnosis, according to Table $1^{15,22}$.

It is important to determine whether sarcoidosis occurs as a systemic disease or if it is restricted to the CNS. For that purpose, one might use the Kveim-Siltzbach procedure, rarely available in Brazil, which consists of an intradermal injection of heat-treated homogenate derived from sarcoid tissue. The development of a noncaseating granuloma at the injection site constitutes a positive response, but the sensitivity is limited, between 60 to $90 \%^{3}$. Chest radiography, thoracic computer tomography scan, and pulmonary function tests may reveal intra-thoracic involvement, which is found in up to $90 \%$ of the patients ${ }^{1}$. Routine laboratory tests may show hyperglobulinemia, hypercalcemia or elevation of alkaline phophatase ${ }^{3}$. Elevated levels of serum angiotensin-converting-enzyme (ACE) are present in 24 to $76 \%$ of the patients ${ }^{23}$. Despite its poor predictive value, ACE remains the only serological marker of sarcoidosis. ACE activity in bronchoalveolar lavage (BAL) is generally considered a good marker of sarcoidosis severity with higher prognostic value than serum

Table 1. Classification of definite, probable, and possible NS.

Possible: the clinical syndrome and neurorradiologic evaluation are suggestive of NS. Infection and malignancy were not rigorously excluded or there is no pathologic confirmation of systemic sarcoidosis

Probable: the clinical syndrome and neurorradiologic evaluation are suggestive of NS and differential diagnoses have been made, especially malignancy and infection. There is pathologic evidence of sarcoidosis

Definite: Probable diagnosis + supportive nervous system pathology or response to therapy for NS over a one- to two-year observation period

NS: neurosarcoidosis. 
$\mathrm{ACE}^{24}$. In addition, BAL can be used as an adjunctive measure to support the diagnosis of sarcoidosis, by demonstrating a cell pattern typical of lymphocytic alveolitis (lymphocyte count greater than $15 \%$ ) and a $\mathrm{T}$-cell $\mathrm{CD}^{+} / \mathrm{CD}^{+}$ratio over 3.5 , although it is present in $50 \%$ of patients ${ }^{22,24}$.

Whole body gallium scan, muscle magnetic resonance imaging, and whole body fluorodeoxyglucose positron emission tomography (PET) are useful approaches to search for systemic sarcoidosis when initial investigation is unrevealing $^{25}$. Usually, obtaining tissue specimens that show noncaseating granulomas best supports the diagnosis of sarcoidosis. Endobronchial ultrasound guided transbronchial needle aspiration of mediastinal lymph nodes has facilitated diagnosis, often eliminating the need for more invasive procedures, such as mediastinoscopy ${ }^{22}$.

For evaluating neurologic manifestations, the preferred imaging technique is MRI. T1-weighted images are useful to evaluate the optic chiasm, hydrocephalus, and spinal cord enlargement. Areas of increased intensity in T2-weighted images and FLAIR can be seen especially in the periventricular distribution. Gadolinium is useful to evaluate leptomeningeal involvement, parenchymal abnormalities (nodular or visual pathway enhancement), and cranial nerve lesions ${ }^{22}$. Fluorodeoxyglucose PET imaging may reveal areas of hyper or hypometabolism within the CNS, which combined with areas of hypermetabolism on systemic PET may be elucidative to NS diagnosis ${ }^{22,24}$. Fig 2 shows an algorithm to investigate NS suspected cases.

CSF analysis can be helpful, although not specific. The majority of patients, particularly those with meningeal involvement, present mononuclear pleocytosis ranging from 10 to 100 cells $/ \mathrm{mm}^{3}$, glucose as low as $30 \mathrm{mg} / \mathrm{dL}$, and elevated

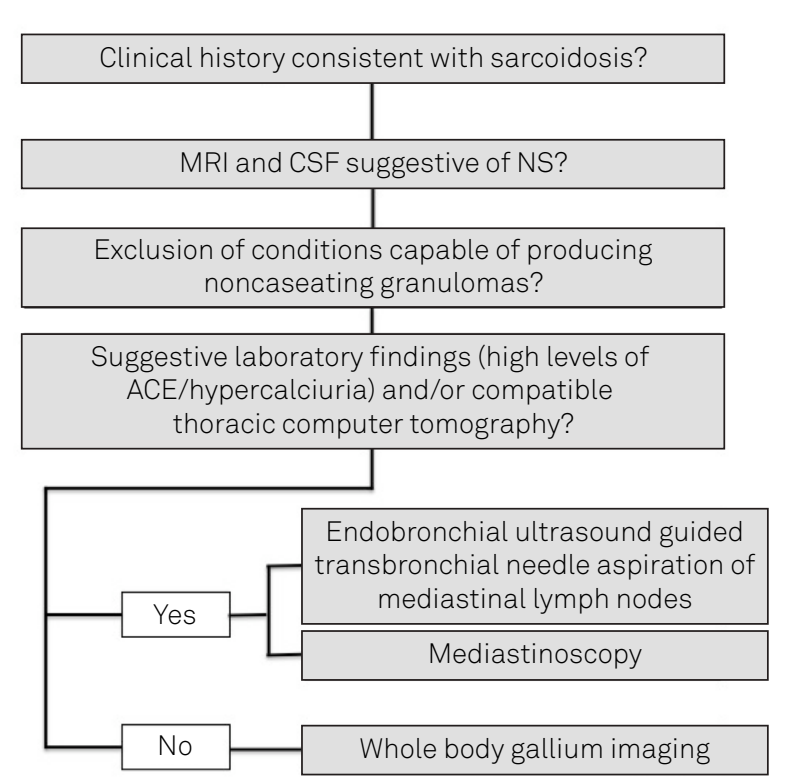

MRI: magnetic resonance image; CSF: cerebral spinal fluid; NS: neurosarcoidosis; ACE: angiotensin converting-enzyme.

Fig 2. Algorithm for investigation of suspected neurosarcoidosis. opening pressure. Elevated CSF IgG titers and oligoclonal bands may be observed in some patients. Although ACE in the CSF is not specific of NS, it is most probably synthesized within the nervous system and seems to be especially useful in the monitoring of disease activity or treatment response ${ }^{1}$. Increased CSF lymphocytes $\mathrm{CD}^{+}$: $\mathrm{CD}^{+}$ratio has also been observed in NS. However, it requires large numbers of viable lymphocytes, which are usually unavailable ${ }^{5}$.

Potential biomarkers to detect disease activity are under investigation. More recently, chitotriosidase, an enzyme involved in the degradation of chitin, may induce overexpression of Th2 cytokines and forecast disease activity and prognosis in sarcoidosis, with sensitivity over $90 \% 5$. IL-2 receptor may also be elevated in BAL of sarcoidosis patients with prognostic value and even higher levels in patients with extrapulmonary involvement $t^{5,19}$.

\section{DIFFERENTIAL DIAGNOSIS}

Differential diagnosis in NS depends on the clinical presentation. The presence of multisystem disease usually helps establishing the diagnosis, especially when pathological analyses showing sarcoid features are available at least from one site. It is important to rule out infectious diseases, neoplasms, and other autoimmune inflammatory disorders. Clinical manifestations and also MRI-based findings may guide the investigation (Table 2).

In the setting of subacute or chronic meningitis with headache, cranial neuropathies and constitutional symptoms, CSF analysis is mandatory. PCR for herpes family viruses have high sensitivity and specificity ${ }^{26}$. On the other hand, PCR for tuberculosis have a variable and otherwise low sensitivity, but high specificity ${ }^{27,28}$. Fungal and tuberculosis (TB) stains and cultures, immunologic analysis for fungal antigens, and oncotic cytology are also important. Thickening and enhancement of the leptomeninges of patients with NS are virtually indistinguishable from those seen in tuberculosis or lymphoma ${ }^{29}$. If dural involvement occurs, one should consider meningioma, dural metastasis, lymphoma, and idiopathic hypertrophic cranial pachymeningitis.

TB and sarcoid meningitis classically have predilection for basal involvement and may cause multiple cranial neuropathies. However, syphilis, lymphoma, viral and fungal meningitis may also involve such region ${ }^{4,30,31}$. When facial palsy, either uni or bilaterally, is present, borreliosis, Fisher and Guillain-Barré syndrome should be considered, as well as HIV infection, mononucleosis, postinfluenza, syphilis, acute porphyria, amyloidosis, and multiple sclerosis ${ }^{4,32}$. The differential diagnosis of isolated optic neuropathy includes optic nerve glioma, optic neuritis or involvement of dural nerve sheath by meningioma ${ }^{15}$. Sarcoidosis may also affect orbital fat, extrinsic ocular muscles or lacrimal glands mimicking orbital pseudotumor ${ }^{33}$. 
Table 2. Differential diagnosis of NS based on clinical and imaging manifestations.

\begin{tabular}{ll} 
Presentation & \multicolumn{1}{c}{ Differential diagnosis } \\
\hline Meningitis & Infections: bacterial, fungal, HIV and other viruses, TB, \\
& syphilis, borreliosis, Whipple's disease \\
& Inflammatory diseases: SLE, BD, SS \\
& Tumors: lymphoma, carcinomatous meningitis \\
& Infections: borreliosis, TB, fungal, syphilis \\
Cranial and/or peripheral neuropathy & Tumors: meningioma, leptomeningeal metastases, gliomas \\
& Inflammatory: Guillain-Barre syndrome, Fisher syndrome, SLE, SS, NBD \\
& Demyelinating: MS \\
& Systemic/Metabolic: amyloidosis, acute porphyria \\
& Infections: syphilis, toxoplasmosis \\
Inflammatory: SLE, SS, NBD & Demyelinating: MS \\
Myelopathy & Infections: viral, fungal \\
& Tumors: meningioma, lymphoma, carcinomatous metastasis, nerve sheath tumors, \\
& chloroma, hemangiopericytoma \\
& Tumors: lymphoma, glioma, metastasis \\
Brain mass lesion & Infections: toxoplasmosis, infectious granulomas \\
& Demyelinating: Tumefactive demyelination \\
& Demyelinating: MS \\
Brain white-matter lesions & Antiphospholipid syndrome \\
Atherosclerotic vascular disease & Inflammatory: SLE, SS, NBD \\
\hline
\end{tabular}

NS: neurosarcoidosis; TB: tuberculosis; SLE: systemic lupus erythematosus; NBD: Neuro-Behçet's disease; SS: Sjoegren syndrome; MS: multiple sclerosis.

Involvement of hypothalamic and pituitary dysfunction in NS may mimic pituitary adenoma, Rathke cleft cyst, and craniopharyngioma. However, polyuria and polydipsia due to diabetes insipidus or evidence of dysfunction of both anterior and posterior pituitary lobes are less frequently associated with tumors ${ }^{32}$. Isolated infundibulum involvement seen as thickening and enhancement on T1-weighted MRI images may also be found in histiocytosis ${ }^{29}$.

Intracranial mass lesions, presenting seizures or focal deficits, should be differentiated from primary and secondary tumors or tumecfative demyelination. In sarcoidosis, enhancing mass lesions are usually associated with adjacent leptomeningeal involvement. Hypointensity on NS lesions T2-weighted images may also be found in lymphoma or hypercellular metastases. Central necrosis is more commonly seen in neoplasms $\mathrm{s}^{4,9,29}$.

\section{TREATMENT}

Isolated cranial nerve abnormalities and aseptic meningitis are frequent monophasic presentations and are at low risk for progression, with the exception of being occasional cases of progressive optic neuropathy ${ }^{34,35}$. Such manifestations usually present good response to short steroid course. However, patients who have parenchymal disease, seizures, mass lesions, leptomeningeal involvement with multiple cranial nerve abnormalities, spinal cord disease, and hydrocephalus often experience a chronic remitting-relapsing course ${ }^{36}$. The development of hydrocephalus due to chronic meningitis may be an ominous sign ${ }^{37}$. These severe manifestations often require high dose and prolonged course of steroids ${ }^{38}$.
Although steroids have been the initial option, many patients require alternative treatments due to steroid intolerance or persistent disease activity. Three studies that assessed the response to steroids alone showed that less than $40 \%$ of the patients stabilized or improved ${ }^{5,15,37,38}$. The report by Scott et al. ${ }^{37}$ selected patients for early aggressive immunosuppressive therapy if they "presented with severe CNS (intracranial lesions, hydrocephalus, myelopathy, seizures, or encephalopathy).' Among the patients selected to receive steroids alone, 35\% improved on this regimen, whereas 69\% improved with immunosuppressive agents. Given the reports of treatment failure with steroid therapy in many patients and the toxic effects associated with long-term steroid use, centers with experience in treating NS often select patients with severe disease for treatment with steroids and alternative immunosuppressive agents (combination therapy) at the time of initial diagnosis ${ }^{36-38}$.

We reviewed the most common prescribed agents for the treatment of NS and proposed a treatment strategy based on current studies from different centers specialized in sarcoidosis and on our own experience (Fig 3).

\section{Steroids}

Steroids remain the mainstay of treatment for $\mathrm{NS}^{5,15,36,37,39}$. They are most useful early in the treatment since they can lead to rapid reduction of the inflammation and the mass effect $^{36}$. For severe manifestations, steroids are started at $1 \mathrm{mg} / \mathrm{kg}$ or as a pulse of methylprednisolone $1,000 \mathrm{mg} /$ day for three days, followed by tapering. For myopathy, facial palsy, and neuropathy, they are started at $0.5 \mathrm{mg} / \mathrm{kg}$ and maintained from two to four weeks, followed by tapering. Patients should be monitored for development of diabetes, 


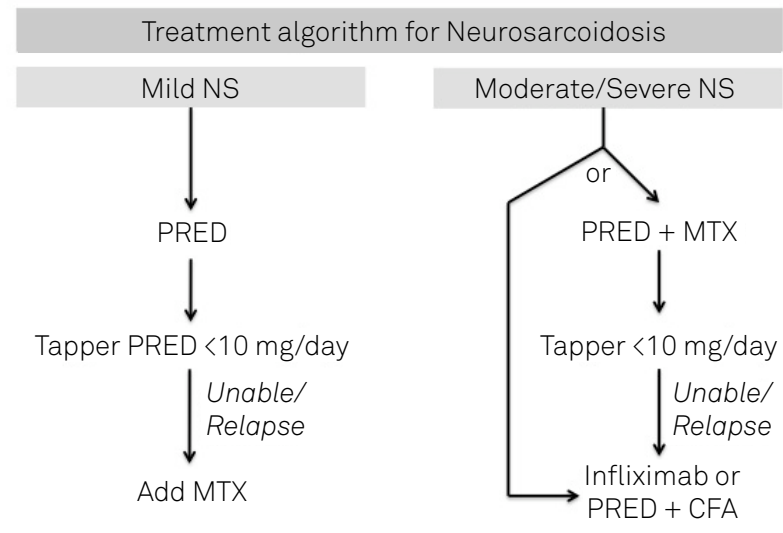

NS: neurosarcoidosis; PRED: prednisone; MTX: methotrexate; CFA: cyclophosphamide.

Fig 3. Suggested treatment strategy for neurosarcoidosis.

glucose intolerance, dislipemia, hypertension, and osteoporosis and frequently low steroids doses are required for more than one year. The goal is to maintain the patients with doses $<10 \mathrm{mg} /$ day $^{40}$.

\section{Immunosuppressive agents}

Methotrexate (MTX), azathioprine (AZA), and mycophenolate (MMF) are immunosuppressive agents commonly prescribed and equally effective. There are no randomized trials defining the optimal treatment for NS. MTX is the most common prescribed immunosuppressant, usually at 7.5 to $15 \mathrm{mg} / \mathrm{kg}$ per week, followed by folic acid supplementation and requires bimonthly evaluation of liver function and blood cell counts with differential. AZA is prescribed at 150 to $200 \mathrm{mg}$ /day, and requires monthly liver function tests and blood cell counts.

MMF is effective in treating cutaneous and renal sarcoidosis. It is associated with less incidence of neutropenia than other cytotoxic agents; however, nausea and diarrhea can be dose-limiting adverse effects ${ }^{40}$. It is prescribed at 1,000 mg twice daily and it is occasionally used in association with infliximab ${ }^{41}$. Although it is considered as a therapeutic option in NS, MMF is not effective in all NS manifestations, presenting low response for myopathy ${ }^{21}$.

Cyclophosphamide (CFA) is reserved for refractory cases, according to previous studies ${ }^{36,38,42,43}$. It is started at $0.5 \mathrm{mg} / \mathrm{m}^{2}$ until a maximum of $1,000 \mathrm{mg}$ in monthly pulses for 6 to 12 months. Liver, renal function, blood cells count should be monitored monthly. CFA is associated with opportunistic and fungal infections, hemorrhagic cystitis, bone marrow aplasia, and higher cancer rates.

\section{Anti-TNF agents}

Infliximab is a chimeric monoclonal antibody that neutralizes the biological activity of TNF-alpha by binding to its soluble and transmembrane forms and inhibiting receptor binding ${ }^{44}$. Evidence for its use came from the finding of elevated TNF-alpha levels in lymph nodes and BAL of patients with sarcoidosis ${ }^{39}$ and by the demonstration that it is a crucial cytokine in the establishment and maintenance of inflammation in several autoimmune disorders ${ }^{44}$. There are 23 reports on the use of infliximab for refractory NS, sometimes in association with other immunosuppressive agents, such as CFA or $\mathrm{MMF}^{36,41,45,46}$. It is prescribed at $5 \mathrm{mg} / \mathrm{kg}$ at zero, two and six weeks and then repeated every eight weeks. Because infliximab may be associated with reactivation of tuberculosis, chest X-rays and tuberculin tests are required before initiating therapy. Infliximab may also precipitate oncological and autoimmune complications, and it was also associated with high mortality rates in patients with advanced congestive heart failure ${ }^{40,47}$.

Etarnecept, which is another TNF-alpha antagonist, presents lower response than infliximab for the treatment of sarcoidosis. The reason for the enhanced efficacy of infliximab is not well understood, but it is believed that infliximab is highly specific for TNF-alpha, whereas etanercept also binds a related molecule, lymphotoxin- $\alpha$ (formerly known as TNFbeta). The molecular structure (an IgG antibody) of infliximab allows it to initiate the classical complement pathway and causes cell lysis, whereas etanercept, a recombinant molecule attached to the Fc portion of human IgG1, cannot. Moreover, infliximab has been demonstrated to induce T-cell apoptosis, and etanercept has not ${ }^{47}$.

Infliximab is a rescue option for patients resistant to CFA and there is evidence that it could be more effective than $\mathrm{CFA}^{42}$. For that reason, some specialists initiate infliximab before CFA for severe manifestations, which have already failed one immunosuppressive agent ${ }^{36,40,44}$.

\section{PROGNOSIS}

Although treatment with steroids can improve MRI, clinical responses may not correlate with radiological improvement ${ }^{36}$. Mortality rates were $18 \%$ in a series of 37 patients and $31 \%$ developed steroid side effects, related to the high dose of steroids and longer duration of therapy ${ }^{48}$. In another series of 54 patients, $10 \%$ developed progressive symptoms despite immunosuppressive treatment ${ }^{49}$.

\section{CONCLUSIONS}

The diagnosis and management of NS remain challenging aspects of the disease. Most cases require immunosuppressive treatment due to the high rates of recurrence or incomplete response. Thus, immunosuppressive agents should be strongly considered at initial treatment. Biological agents such as infliximab are good options in treating refractory cases. 


\section{References}

1. Hoitsma E, Faber CG, Drent M, Sharma OP. Neurosarcoidosis: a clinical dilemma. Lancet Neurol 2004;3:397-407.

2. Sharma OP, Shigemitsu H.A historical sketch: Life and time of Jonathan Hutchinson (1828-1913), the first sarcoidologist. Sarcoidosis Vasc Diffuse Lung Dis 2008;25:71-75.

3. Vinas FC, Rengachary S. Diagnosis and management of neurosarcoidosis. J Clin Neurosci 2001;8:505-513.

4. Nowak DA, Widenka DC. Neurosarcoidosis: a review of its intracranial manifestation.J Neurol 2001;248:363-372.

5. LowerEE,BroderickJP,BrottTG,Baughman RP.Diagnosisand management of neurological sarcoidosis. Arch Intern Med 1997;157:1864-1868.

6. Whelan MA, Stern J.Sarcoidosis presenting as a posterior fossa mass. Surg Neurol 1981;15:455-457.

7. Tsao CY, Lo WD, Rusin JA, Henwood MJ, Boue DR. Isolated neurosarcoidosis presenting as headache and multiple brain and spinal cord lesions mimicking central nervous system metastases. Brain Dev 2007;29:514-518.

8. Sponsler JL, Werz MA, Maciunas R, Cohen M. Neurosarcoidosis presenting with simple partial seizures and solitary enhancing mass: case reports and review of the literature. Epilepsy Behav 2005;6:623-630.

9. Chapelon C, Ziza JM, Piette JC, et al. Neurosarcoidosis: signs, course and treatment in 35 confirmed cases. Medicine (Baltimore) 1990;69:261-276.

10. Lawrence WP, el-Gammal T, Pool WH, Jr, Apter L. Radiological manifestations of neurosarcoidosis: report of three cases and review of literature. Clin Radiol 1974;25:343-348.

11. Oksanen V. Neurosarcoidosis: clinical presentations and course in 50 patients. Acta Neurol Scand 1986;73:283-290.

12. Byrne JV, Lawton CA. Meningeal sarcoidosis causing intracranial hypertension secondary to dural sinus thrombosis. Br J Radiol 1983;56:755-757.

13. Gilmore K, Rudden M, Kalman TP. Psychiatric manifestations of sarcoidosis. Can J Psychiatry 1980;25:329-331.

14. Scott TF. Neurosarcoidosis: progress and clinical aspects. Neurology 1993;43:8-12.

15. Zajicek JP, Scolding NJ, Foster O, et al. Central nervous system sarcoidosis-diagnosis and management. QJM 1999;92:103-117.

16. Delaney P. Neurologic manifestations in sarcoidosis: review of the literature, with a report of 23 cases. Ann Intern Med 1977;87:336-345.

17. Vital A, Lagueny A, Ferrer X, Louiset P, Canron MH, Vital C. Sarcoid neuropathy: clinico-pathological study of 4 new cases and review of the literature. Clin Neuropathol 2008;27:96-105

18. Chen ES, Moller DR. Etiology of sarcoidosis. Clin Chest Med 2008:29:365-377.

19. Morgenthau AS, lannuzzi MC. Recent advances in sarcoidosis. Chest $2011: 139 \cdot 174-182$

20. Newman LS, Rose CS, Bresnitz EA, et al. A case control etiologic study of sarcoidosis: environmental and occupational risk factors. Am J Respir Crit Care Med 2004;170:1324-1330.

21. Gerke AK, Hunninghake G. The immunology of sarcoidosis. Clin Chest Med. 2008:29:379-390.

22. Stern BJ. Neurological complications of sarcoidosis. Curr Opin Neurol 2004;17:311-316.

23. Lynch JP 3rd. Neurosarcoidosis: how good are the diagnostic tests? J Neuroophthalmol 2003:23:187-189.

24. Bargagli E, Mazzi A, Rottoli P. Markers of inflammation in sarcoidosis: blood, urine, BAL, sputum, and exhaled gas. Clin Chest Med. 2008;29:445-458

25. Dubey N, Miletich RS, Wasay M, Mechtler LL, Bakshi R. Role of fluorodeoxyglucose positron emission tomography in the diagnosis of neurosarcoidosis. J Neurol Sci 2002;205:77-81.
26. Lakeman FD, Whitley RJ. Diagnosis of herpes simplex encephalitis: application of polymerase chain reaction to cerebrospinal fluid from brain-biopsied patients and correlation with disease. National Institute of Allergy and Infectious Diseases Collaborative Antiviral Study Group. J Infect Dis 1995;171:857-863.

27. Donald PR, Victor TC, Jordaan AM, Schoeman JF, van Helden PD. Polymerase chain reaction in the diagnosis of tuberculous meningitis. Scand J Infect Dis 1993;25:613-617.

28. Nguyen LN, Kox LF, Pham LD, Kuijper S, Kolk AH. The potential contribution of the polymerase chain reaction to the diagnosis of tuberculosis meningitis. Arch Neurol 1996;53:771-776.

29. Smith JK, Matheus MG, Castillo M. Imaging manifestations of neurosarcoidosis. Am J Roentgenol 2004;182:289-295.

30. Stern BJ, Krumholz A, Johns C, Scott P, Nissim J. Sarcoidosis and its neurological manifestations. Arch Neurol 1985;42:909-917.

31. Ranoux D, Devaux B, Lamy C, Mear JY, Roux FX, Mas JL. Meningeal sarcoidosis, pseudo-meningioma, and pachymeningitis of the convexity. J Neurol Neurosurg Psychiatry 1992;55:300-303.

32. Hoitsma E, Drent M, Sharma OP. A pragmatic approach to diagnosing and treating neurosarcoidosis in the 21st century. Curr Opin Pulm Med 2010:16:472-479.

33. Carmody RF, Mafee MF, Goodwin JA, Small K, Haery C. Orbital and optic pathway sarcoidosis: MR findings. AJNR Am J Neuroradiol 1994;15:775-783

34. Ferriby D, de Seze J, Stojkovic T, et al. Long-term follow-up of neurosarcoidosis. Neurology 2001;57:927-929.

35. Chapelon-Abric C, Ziza JM, Piette JC, et al. Neurosarcoidosis. Ann Med Interne (Paris) 1991;142:601-608.

36. Lower EE, Weiss KL. Neurosarcoidosis. Clin Chest Med 2008;29:475-492.

37. ScottTF, Yandora K, Valeri A, Chieffe C, Schramke C. Aggressive therapy for neurosarcoidosis: long-term follow-up of 48 treated patients. Arch Neurol 2000;64:691-696.

38. Agbogu BN, Stern BJ, Sewell C, Yang G. Therapeutic considerations in patients with refractory neurosarcoidosis. Arch Neurol 1995;52:875-879.

39. Hunninghake GW, CostabelU,Ando M, etal.ATS/ERS/WASOG statement on sarcoidosis. American Thoracic Society/European Respiratory Society/World Association of Sarcoidosis and other Granulomatous Disorders. Sarcoidosis Vasc Diffuse Lung Dis 1999;16:149-173.

40. Baughman RP, Costabel U, du Bois RM. Treatment of sarcoidosis. Clin Chest Med 2008;29:533-548.

41. Moravan M, Segal BM. Treatment of CNS sarcoidosis with infliximab and mycophenolate mofetil. Neurology 2009;72:337-340.

42. Doty JD, Mazur JE, Judson MA. Treatment of corticosteroid-resistant neurosarcoidosis with a short-course cyclophosphamide regimen. Chest 2003;124:2023-2026.

43. Vargas DL, Stern BJ. Neurosarcoidosis: diagnosis and management Semin Respir Crit Care Med 2010;31:419-427.

44. Santos E, Shaunak S, Renowden S, Scolding NJ. Treatment of refractory neurosarcoidosis with Infliximab. J Neurol Neurosurg Psychiatry 2010;81:241-246.

45. Pettersen JA, Zochodne DW, Bell RB, Martin L, Hill MD. Refractory neurosarcoidosis responding to infliximab. Neurology 2002;59:1660-1661.

46. Pereira J, Anderson NE, McAuley D, Bergin P, Kilfoyle D, Fink J. Medically refractory neurosarcoidosis treated with infliximab. Intern Med J 2011;41:354-357

47. Doty JD, Mazur JE, Judson MA. Treatment of sarcoidosis with infliximab. Chest 2005;127:1064-1071.

48. Om P. Sharma. Neurosarcoidosis: A Personal Perspective Based on the Study of 37 Patients. Chest 1997;112:220-228.

49. Pawate S, Moses H, Sriram S. Presentations and outcomes of neurosarcoidosis: a study of 54 cases. QJM Med 2009;102:449-460. 